\title{
Traumatische Erlebnisse in der Kindheit und psychiatrische Komorbidität bei erwachsenen männlichen Alkoholkranken: Empirische Untersuchungsergebnisse und ihre klinische Bedeutung
}

\author{
W. Langeland ${ }^{1}$ \\ W. van den Brink ${ }^{2}$ \\ N. Draijer ${ }^{3}$
}

\author{
Childhood Trauma and Psychiatric Co-Morbidity in Adult Male Alcoholics: \\ Empirical Findings and Clinial Implications
}

\section{Zusammenfassung}

Im Rahmen dieses Beitrags untersuchten wir mögliche Zusammenhänge zwischen traumatischen Erlebnissen in der Kindheit und komorbiden psychischen Störungen, wie sie üblicherweise bei männlichen Alkoholpatienten gefunden werden, die nicht $\mathrm{zu}$ Stichproben von Kriegsveteranen gehören. Es werden einerseits Ergebnisse bisheriger Studien im Überblick vorgestellt, zum anderen auch einige neue Ergebnisse unserer eigenen Untersuchungen. Befunde zum Zusammenhang zwischen Traumatisierungen in der Kindheit und Achse-I- bzw. Achse-II-Komorbidität zeigen ein breites Spektrum psychiatrischer Diagnosen bei männlichen Alkoholpatienten, die Viktimisierungserfahrungen in der Kindheit berichten, insbesondere Dysthymie, Angststörungen und Posttraumatische Belastungsstörungen (PTBS). Darüber hinaus deuten die vorhandenen Daten darauf hin, dass elterlicher Alkoholismus, das Miterleben von elterlicher Gewalt sowie physische Misshandlung eine Rolle für die spätere Entwicklung einer antisozialen Persönlichkeitsstörung bei männlichen Alkoholkranken spielen. Die in der klinischen Versorgung Tätigen sollten daher in Bezug auf zusätzliche psychiatrische Störungen bei männlichen Patienten, die wegen Alkoholabhängigkeit zur Behandlung kommen, besonders aufmerksam sein. Diagnostische Probleme und Behandlungsansätze werden diskutiert.

\section{Schliuisselwörter}

Kindliche Traumatisierungen - männliche Alkoholpatienten • psychiatrische Komorbidität

\section{Abstract}

In this article we examined possible relations between childhood trauma and diagnostic co-morbidity typically seen in non-veteran male alcoholic patients. In addition to reviewing results of previous studies, we also present some new findings based on our own research. Findings on the relationship between childhood trauma and Axis I and II co-morbidity indicate extensive diagnostic heterogeneity in male alcoholic patients reporting childhood victimization, in particular dysthymia, phobic disorders and posttraumatic stress disorder (PTSD). Furthermore, existing data suggest a role for parental alcoholism, witnessing parental violence, and physical abuse in predicting antisocial personality disorder in male alcoholic patients. Clearly, clinicians need to be aware of the presence of trauma and concomitant psychiatric disorders, including PTSD among male patients seeking alcohol addiction treatment. Diagnostic issues as well as treatment approaches are discussed.

Key words

Childhood trauma $\cdot$ male alcoholic patients $\cdot$ psychiatric comorbidity

${ }^{1}$ Amsterdam Institute for Addiction Research, Department of Psychiatry of the Vrije Universiteit Amsterdam

${ }^{2}$ Amsterdam Institute for Addiction Research, Academic Medical Center of the University of Amsterdam

${ }^{3}$ Department of Psychiatry of the Vrije Universiteit Amsterdam

Korrespondenzadresse

Dr. Willie Langeland·Quartier Mounicat · 32190 Bascous · Frankreich ·E-mail: gjwil.langdijk@wanadoo.fr

Bibliografie

Suchttherapie 2004; 5: 132 - 136 @ Georg Thieme Verlag KG Stuttgart · New York

DOI 10.1055/s-2004-813528

ISSN 1439-9903 
Einleitung

Sowohl Ärzten wie Wissenschaftlern ist bekannt, dass Alkoholabhängigkeit häufig zusammen mit anderen psychiatrischen Störungen auftritt. Eines der aktuellen theoretischen Modelle erklärt das Phänomen der Komorbidität mit gemeinsamen ätiologischen Faktoren, wie genetischer Prädisposition oder milieubedingten Risiken. Bezüglich milieubedingter Risikofaktoren zeigen Untersuchungen an klinischen Stichproben von Alkoholpatienten, dass sie in weitaus größerem Ausmaß als die Allgemeinbevölkerung traumatischen Erlebnissen in der Kindheit ausgesetzt waren. Andererseits wurden bei Erwachsenen mit frühen traumatischen $\mathrm{Er}$ lebnissen in hohem Maße Alkoholmissbrauch und andere psychiatrische Störungen festgestellt [1 - 3].

Es wurde angenommen, dass das höhere Risiko eines schlechteren Behandlungsergebnisses bei Personen mit Alkoholproblemen und körperlichen Misshandlungen oder sexuellem Missbrauch in der Vorgeschichte auf ein größeres Ausmaß psychiatrischer Probleme, mangelnde soziale Unterstützung und Schwierigkeiten, sich auf eine therapeutische Beziehung einzulassen, zurückzuführen ist (vgl. [4]). Weiter nahm man an, dass schlechte Behandlungsergebnisse eher mit komorbiden Posttraumatischen Belastungsstörungen (PTBS) als mit psychiatrischer Komorbidität im Allgemeinen in Verbindung stehen [5]. Eine PTBS scheint dabei eine besonders große Rolle bei alkohol- und drogenabhängigen Frauen zu spielen, die in der Kindheit oder als Erwachsene sexuellem Missbrauch und/oder körperlicher Misshandlung ausgesetzt waren [6]. Andererseits zeigte sich in einer deutschen Multizenterstudie, dass komorbide Belastungsstörungen bei alkoholabhängigen Patienten den Verlauf und den Schweregrad der Alkoholabhängigkeit unabhängig vom Geschlecht beeinflussen. So berichteten Alkoholpatienten mit PTBS einen früheren Trinkbeginn und einen früheren Beginn von Entzugssymptomen und hatten mehr Entzugsbehandlungen gemacht als Patienten ohne PTBS [7].

\section{Fehlende Daten zu männlichen Alkoholpatienten}

Verschiedene der oben beschriebenen klinischen Beobachtungen wurden durch empirische Studien bei weiblichen Alkoholpatientinnen und bei männlichen Kriegsveteranen mit Alkoholabhängigkeit bestätigt [4]. Allerdings wurde der Zusammenhang zwischen sexuellen und körperlichen Traumatisierungen in der Kindheit und psychiatrischer Komorbidität bei der Patientengruppe, die im klinischen Alltag überwiegt, nämlich männlichen Patienten, die keine Kriegsveteranen sind, bislang nicht ausführlicher untersucht. Bezüglich der Art der komorbiden psychiatrischen Störung wurden Geschlechtsunterschiede nachgewiesen, zum Beispiel eine höhere durchschnittliche Anzahl von Achse-I-Störungen bei Frauen und eine niedrigere Prävalenz von Lebenszeit- und gegenwärtiger PTBS bei Männern (z. B. [8, 9]). Bei klinischen Stichproben von männlichen Alkoholpatienten finden sich neben Angststörungen (insbesondere Phobien) und affektiven Störungen (insbesondere schweren Depressionen) häufig antisoziale Persönlichkeitsstörungen [10]. Dies wirft die Frage auf, ob traumatische Kindheitserlebnisse tatsächlich zu der für männliche Alkoholkranke typischen Komorbidität beitragen. Falls dies der Fall sein sollte, stellte sich die Frage, welche zusätzlichen psychiatrischen Störun- gen genau bei der Subgruppe von Patienten mit (wiederholtem) Missbrauch in der Kindheit zu finden sind.

Ziel dieser Arbeit ist, Informationen zum klinischen Profil von männlichen Alkoholkranken mit frühen physischen/sexuellen Traumatisierungen zur Verfügung zu stellen. Hierbei beziehen wir uns ausdrücklich nur auf die Ergebnisse der Untersuchungen an männlichen Patienten, die nicht der Gruppe der Kriegsveteranen angehören. Zunächst geben wir dazu einen kurzen Überblick über Befunde zu den Prävalenzraten von körperlicher Misshandlung und sexuellem Missbrauch in klinischen Stichproben von männlichen Alkoholpatienten sowie zur Prävalenz weiterer widriger Umstände in der Lebensgeschichte dieser Patienten. Anschließend wird der derzeitige Wissensstand zur Bedeutung von kindlichen Traumatisierungen und für die Achse-I-Komorbidität bei männlichen Alkoholkranken kurz umrissen. Weiter werden die vorhandenen Daten zum Zusammenhang zwischen Kindheitstraumata und Achse-II-Komorbidität bei erwachsenen männlichen Alkoholpatienten vorgestellt. Abschließend diskutieren wir die Implikationen dieser Befunde für die Diagnostik und Behandlung.

Einige der hier dargestellten Ergebnisse basieren auf eigenen, bereits publizierten wissenschaftlichen Daten [11 - 15]. Der vorliegende Artikel enthält jedoch auch bislang unveröffentlichte Ergebnisse. Sie betreffen eine Stichprobe von 122 männlichen Patienten mit Alkoholabhängigkeit, die bereit waren, an einer Studie zu psychiatrischer Komorbidität und Behandlungsoutcome teilzunehmen, und einer konsekutiven Gruppe von Patienten entstammten, die in einem Suchttherapiezentrum in der Region von Amsterdam (Niederlande) um Behandlung gebeten hatten. Zur Exploration von Traumatisierungen und Vernachlässigung in der Kindheit wurde das Strukturierte Trauma Interview (STI) eingesetzt [16]. ${ }^{1}$ Eine ausführliche Beschreibung des Studiendesigns und weitere relevante Informationen finden sich bei Langeland et al. [15].

Kindliche Traumatisierungen und andere belastende Lebensumstände bei männlichen Alkoholpatienten mit Behandlungswunsch

Männliche Alkoholpatienten berichten häufig über frühe Viktimisierungserfahrungen. Bei klinischen Stichproben männlicher Alkoholpatienten wurden Prävalenzraten kindlichen sexuellen Missbrauchs von 12\% [17] bzw. 18\% [15] gefunden. Die beobachtete Häufigkeit von körperlicher Misshandlung in der Kindheit lag zwischen 13 und 31 \% (vgl. [2, 15]), wobei in Studien, in denen die Definition für körperliche Misshandlung enger gefasst worden war, niedrigere Raten gefunden wurden. Die Prävalenzrate für das gemeinsame Vorliegen beider Formen beträgt bei männlichen Alkoholpatienten 5\% [15, 17]. Verglichen mit weiblichen Patientinnen, berichten männliche Alkoholpatienten häufiger lediglich über körperliche Misshandlung in der Kindheit.

\footnotetext{
1 Das STI wurde bereits in verschiedenen Forschungsprojekten und in der klinischen Diagnostik eingesetzt. Bislang wurden keine nachteiligen Folgen seiner Anwendung beobachtet. Das Interview wurde vor kurzem ins Deutsche und Französische übersetzt.
} 
Darüber hinaus deuten neuere Daten bei männlichen Alkoholpatienten $(n=122)$ auf eine signifikant erhöhte Rate auch anderer belastender Lebensumstände bei Patienten hin, die über körperlichen Missbrauch durch elterliche Personen in der Kindheit berichten. So fand sich bei dieser Gruppe von Patienten eine 14fach erhöhte Rate des Miterlebens von Gewalt zwischen den Eltern während der Kindheit, eine 4fach erhöhte Rate von Alkoholproblemen der Mutter, eine 9fach erhöhte Rate von Alkoholproblemen des Vaters und eine fast 7 fach erhöhte Rate für sexuelle Viktimisierung im Erwachsenenalter. Aus klinischer Sicht ist dabei die Erkenntnis wichtig, dass ein starker Zusammenhang zwischen einem gewalttätigen häuslichen Umfeld und Alkoholproblemen der Eltern gefunden wurde. Dieser Umstand ist deshalb von Bedeutung, weil Zusammenhänge zwischen kindlicher Misshandlung und psychiatrischer Komorbidität lediglich das Resultat anderer, häufig in einem aggressiven Entwicklungsumfeld vorkommender Faktoren sein könnten (z. B. [18]).

\section{Traumatisierungen in der Kindheit und}

Achse-I-Komorbidität bei männlichen Alkoholpatienten mit Behandlungswunsch

Die vorliegenden Ergebnisse deuten darauf hin, dass sexueller Missbrauch und/oder körperliche Misshandlung in der Kindheit mit einer erhöhten Rate von komorbiden affektiven Störungen und Angststörungen sowie suizidalem Verhalten bei männlichen Alkoholpatienten in Behandlung einhergehen [15, 17]. Windle et al. (1995) fanden in ihrer Studie Zusammenhänge zwischen sexuellem Missbrauch bzw. körperlicher Misshandlung und höheren Raten von Suizidversuchen und schweren Depressionen bei männlichen Patienten. Besonders aussagekräftig sind auch die Befunde unserer eigenen Studie, die zeigten, dass Traumatisierungen in der Kindheit (besonders sexueller Missbrauch) bei männlichen wie weiblichen Alkoholabhängigen mit Behandlungswunsch zu einer erhöhten Komorbidität beitrugen, und zwar unabhängig von weiteren belastenden Kindheitserlebnissen (früher Verlust der Eltern, Miterleben von Gewalt zwischen den Eltern, Alkoholkrankheit und/oder andere elterliche Störungen) und Viktimisierung im Erwachsenenalter [15]. Bei männlichen Patienten mit frühen Traumatisierungen zeigte sich dies in der höheren durchschnittlichen Anzahl komorbider phobischer Störungen und PTBS. Außerdem wiesen männliche Alkoholkranke, die schwerere und intrusivere Formen von frühem sexuellen Missbrauch sowie multiple frühe Traumatisierungen berichteten, komplexere Symptomkonstellationen auf, die Dysthymie, soziale Phobie und Agoraphobie sowie PTBS einschlossen. Von den Männern in unserer Stichprobe von Alkoholpatienten in Behandlung erfüllten $14 \%$ die diagnostischen Kriterien einer Lebenszeit-PTBS und 5\% die einer aktuellen PTBS. Das zuletzt genannte Ergebnis deckt sich mit den $6 \%$ aktueller PTBS, die in Norwegen bei einer Stichprobe von männlichen Alkoholpatienten in Behandlung gefunden wurden [9]. Beide Studien setzten das Composite International Diagnostic Interview (CIDI) ein. Schließlich zeigen unsere Ergebnisse, dass nicht nur Misshandlung und Missbrauch in der Kindheit, sondern auch andere belastende Umstände im Kindes- wie im Erwachsenenalter für das Verständnis von psychiatrischer Komorbidität bei männlichen Alkoholpatienten von Bedeutung sind [15].
Traumatisierungen in der Kindheit und antisoziale Persönlichkeitsstörung bei männlichen Alkoholpatienten mit Behandlungswunsch

Das klinische Profil von Alkoholpatienten in Behandlung wird durch das häufige Vorliegen von Persönlichkeitsstörungen, bei männlichen Alkoholkranken insbesondere die antisoziale Persönlichkeitsstörung (ASP), kompliziert. Generell ist in der klinischen Praxis eine Komorbidität in Form von Achse-I- und Achse-II-Störungen eher die Regel als die Ausnahme. Achse-II-Störungen gehen dabei auch mit Schwierigkeiten in der therapeutischen Beziehung, Non-Compliance und vorzeitigem Therapieabbruch einher. Weiter zeigen wissenschaftliche Befunde, dass viele Patienten mit Persönlichkeitsstörungen Misshandlung oder Vernachlässigung in der Kindheit berichten. Am deutlichsten wurden diese Zusammenhänge durch eine prospektive Studie belegt, die zeigt, dass Personen mit dokumentierten Misshandlungen in der Kindheit ein höheres Risiko für spätere Persönlichkeitsstörungen haben, unabhängig von anderen Risikofaktoren [19]. Insbesondere waren dokumentierte körperliche Misshandlung und Vernachlässigung mit Symptomen der ASP assoziiert.

Der Zusammenhang zwischen Traumatisierungen in der Kindheit und komorbiden Achse-II-Störungen bei männlichen Alkoholpatienten ist bislang nicht ausreichend untersucht worden. Derzeit sind nur Daten zum Zusammenhang zwischen frühen Traumatisierungen und der ASP verfügbar. Die publizierten Daten deuten auf folgende Zusammenhänge hin: Männliche Alkoholiker mit ASP, die zur Behandlung kommen, berichten häufiger als männliche Alkoholiker ohne ASP, dass sie Zeuge elterlicher Gewalt waren (52 versus 16\%), in der Kindheit körperlich oder emotional misshandelt wurden (38 versus $11 \%$ ), sowie über Alkoholismus in der Familie (89 versus 68\%) [20]. Weitere Befunde zeigen, dass sowohl alleinige physische Misshandlung als auch alleiniger sexueller Missbrauch als auch das gemeinsame Vorliegen beider Formen signifikante Prädiktoren für ASP bei stationär behandelten männlichen Alkoholpatienten sind [17]. Alkoholismus in der Familie war in dieser Studie ebenfalls ein signifikanter Prädiktor für ASP. Diese Ergebnisse bestätigen frühere Befunde, die auf die Bedeutung von elterlichem Alkoholismus und körperlichem Missbrauch bei der Entwicklung von antisozialem Verhalten hinwiesen [z. B. 18].

Neue Ergebnisse unserer Studie zeigen, dass 17\% der männlichen Alkoholkranken die DSM-III-R-Kriterien für antisoziale Persönlichkeitsstörung erfüllten. Obwohl körperliche Misshandlung und sexueller Missbrauch in der Kindheit ${ }^{2}$ keinen signifikanten Zusammenhang mit ASP aufwiesen, war dies für das Miterleben von Gewalt zwischen den Eltern ${ }^{3}$ und elterliche Alkoholproble$m^{4}$ der Fall. Nach Kontrolle von konfundierenden Effekten im Hinblick auf andere widrige Kindheitserlebnisse und Erlebnisse körperlicher Gewalt im Erwachsenenalter waren Alkoholprobleme der Mutter besonders bedeutsam für das Vorliegen einer ASP $^{5}$. Ein Trend zur Signifikanz zeigte sich in Bezug auf das Mit-

\footnotetext{
2 Körperliche Misshandlung in der Kindheit bei 25\% der ASP-Gruppe und $12 \%$ der Nicht-ASP-Gruppe $(\mathrm{p}<.10)$

$3 \chi^{2}=5,52, \mathrm{p}<, 05$

4 Mutter: $\chi^{2}=8,44, \mathrm{p}<, 01 /$ Vater: $\chi^{2}=4,08, \mathrm{p}<, 05$

$5 ß=1,73, p<, 01$
} 
erleben von Gewalt zwischen den Eltern ${ }^{6}$. Darüber hinaus wurde ein signifikanter Zusammenhang zwischen einer PTBS in der Folge von Kindheitserlebnissen und der ASP festgestellt: 30\% der männlichen Alkoholpatienten mit ASP erfüllten auch die diagnostischen Kriterien einer Lebenszeit-PTBS, verglichen mit $12 \%$ der Nicht-ASP-Patienten? ${ }^{7}$. In der klinischen Literatur wurden auch aggressive Symptome und die geringe Empathiefähigkeit bei Untergruppen von männlichen Patienten mit ASP mit Symptomen einer PTBS in der Folge kindlicher Misshandlungen in Zusammenhang gebracht, wie zum Beispiel Alexithymie und Störungen der Impulskontrolle [21].

\section{Diagnostische Probleme}

Die verfügbaren Befunde zum Zusammenhang zwischen kindlichen Traumatisierungen und Achse-I- bzw. Achse-II-Komorbidität bei männlichen Alkoholpatienten in Behandlung zeigen eine große diagnostische Heterogenität bei Personen, die frühe Traumatisierungen berichten. Komplexe klinische Profile wurden auch bei anderen Patientengruppen mit frühen, chronischen und schwerwiegenden Traumatisierungen beobachtet (z. B. [22, 23]) und deuten eher auf komplexe als auf einfache Formen der PTBS bei früh traumatisierten Personen hin [24]. Wenn sie vorliegen, sind PTBS oft in ein komplexes Muster von Psychopathologie eingebettet. Diese komplexe Psychopathologie wird oft nicht als „echte Komorbidität" angesehen, da die verschiedenen Störungen typischerweise Teil einer zeitlichen und ätiologischen Kaskade sind und nicht unabhängig voneinander vorliegen [25]. Diese Ergebnisse bestätigen die klinische Beschreibung der "komplexen PTBS“ von Herman [26], die auch die ausgedehnte Komorbidität bei Patienten mit wiederholten interpersonellen Traumatisierungen und schweren psychischen Störungen berücksichtigt. Die verfügbaren Daten deuten darauf hin, dass Alkoholmissbrauch oder -abhängigkeit ein untrennbarer Bestandteil einer komplexen Problemkonstellation sein könnte, die Viktimisierungserfahrungen in der Kindheit und weitere negative Lebensereignisse bzw. -bedingungen beinhaltet und eine Reihe weiterer psychischer Beeinträchtigungen umfasst.

\section{Diagnostische Fragestellungen}

Kliniker sollten bei männlichen Alkoholpatienten in Bezug auf Traumatisierungen und die begleitende Diagnose einer PTBS besonders aufmerksam sein. Die Ergebnisse zur Häufigkeit von (multiplen) Traumatisierungen sprechen für eine routinemäßige, umfassende Exploration dieser Erfahrungen im Vorfeld der Behandlung, die den komplexen Traumaanamnesen Rechnung trägt. Durch einige kleine Veränderungen könnte die Sensitivität des am häufigsten eingesetzten klinischen Instruments, des „European Addiction Severity Index“, bezüglich von Gewalterfahrungen in der Vorgeschichte männlicher Alkoholkranker verbessert werden $[11,14]$. Darüber hinaus ist die umfassende Erhebung der Psychopathologie einschließlich der Achse-I- und Achse-II-Störungen ein notwendiger erster Schritt, um Patienten mit diesen zusätzlichen Diagnosen adäquat zu versorgen. Screeningfragen zu PTBS sollten

\footnotetext{
$6 \mathrm{p}<, 10$

$\chi^{2}=4,45, p<, 05$
}

in allen Einrichtungen zur Alkoholbehandlung zur Standarddiagnostik gehören. Da die üblichen diagnostischen Testverfahren häufig keine Items zu ihren Symptomen einschließen, muss damit gerechnet werden, dass PTBS zu selten diagnostiziert werden [27]. Inzwischen liegt auch eine Reihe von Instrumenten und Interviews zur Diagnostik von Traumatisierungen vor (siehe z. B. [27]).

Aus klinischer Sicht ist es wichtig festzuhalten, dass die Befunde zu den häufigen diagnostischen Problemen teilweise die Tatsache widerspiegeln könnten, dass PTBS in der Folge von Kindheiterlebnissen, wie in der Praxis häufig beobachtet, ein hohes Maß an psychiatrischer Komorbidität aufweisen [28]. Beispielsweise fanden sich in unserer Stichprobe von männlichen Alkoholpatienten signifikante Zusammenhänge zwischen der Diagnose einer PTBS und der einer sozialen Phobie $(r=, 19, p<, 05)$. Möglicherweise war dies dabei eher auf Symptomüberlappungen zwischen beiden Störungen zurückzuführen (z.B. in Panikattacken und Vermeidung) als auf das Vorliegen zweier getrennter Störungen. In Übereinstimmung mit der Überlegung, die PTBS und ihre Komorbidität eher als ein komplexes Spektrum von Traumafolgen zu sehen, wurden in Stichproben aus der Allgemeinbevölkerung phobische Symptome eher bei chronischer als bei akuter PTBS gefunden (z. B. [29]). Bei Patienten mit sexuellen Traumatisierungen in der Vorgeschichte könnte es sinnvoll sein zu untersuchen, ob soziales Vermeidungsverhalten, das bei beiden Störungen auftritt, eher im Rahmen einer PTBS oder einer sozialen Phobie gesehen werden sollte (s. [30]). Im Hinblick auf die Bedeutung sexueller Traumatisierungen bei männlichen Alkoholkranken für agoraphobische Symptome sollte untersucht werden, ob Ängste oder phobisches Vermeidungsverhalten nicht eher durch Erinnerungsreize verursacht werden bzw. Vermeidung im Rahmen einer posttraumatischen Störung darstellen. Es ist allgemein bekannt, dass männliche Patienten zumeist sexuellen Missbrauch durch Täter außerhalb der Familie berichten.

\section{Behandlung}

Derzeit gibt es keinen systematischen Behandlungsansatz mit nachgewiesener Effektivität für alkoholabhängige Patienten, die unter PTBS als Folge von traumatischen Kindheitserlebnissen leiden. Große Erwartungen werden jedoch in ein kognitiv-behaviorales Behandlungsprogramm ohne Expositionsanteile gesetzt („Seeking Safety“), für das (europäische) Evaluationsstudien aktuell in Vorbereitung sind [31]. Es sollte erwähnt werden, dass alkoholabhängige PTBS-Patienten mit multiplen Traumatisierungen, die viele Jahre vor der Behandlung stattfanden (z.B. schwerer sexueller Missbrauch in der Kindheit), weniger ideale Kandidaten für eine Expositionsbehandlung darstellen [32].

Als Richtlinie für die klinische Praxis wird der derzeitige Behandlungsstandard für früh traumatisierte Patienten im Allgemeinen empfohlen; er legt besonderes Gewicht auf Stabilisierung (Umgang mit akuter Intoxikation/Entzug und psychiatrischen Symptomen) und Symptomreduktion (z.B. [33]). Um vorherrschende Symptome (häufig vegetative Übererregung) zu kontrollieren, könnten diese Patienten zusätzlich von Interventionen profitieren, die sich darauf konzentrieren, die Verschlechterung der Symptomatik während der Entzugsphase zu verringern und das Rückfallrisiko zu reduzieren. Beispiele für solche Interventionen 
sind Psychoedukation (Aufklärung des Patienten über die Symptome and den Zusammenhang zwischen den Symptomen und den Alkoholproblemen) und das Training von Copingstrategien (Förderung alternativer Verhaltensweisen zum Umgang mit Stress und Angst). Methoden der allgemeinen Rückfallprophylaxe könnten in Bezug auf potenzielle traumabedingte Rückfallsituationen ergänzt werden.

\section{Literatur}

${ }^{1}$ Van der Kolk BA. The complexity of adaptation to trauma. In: Van der Kolk BA, McFarlane AC, Weisaeth L (Hrsg). Traumatic stress. The effects of overwhelming experience on mind, body, and society. New York: The Guilford Press, 1996: 182-213

${ }^{2}$ Langeland W, Hartgers C. Child sexual and physical abuse and alcoholism: A review. J Stud Alcohol 1998; 59: 336 -348

${ }^{3}$ Simpson TL, Miller WR. Concomitance between childhood sexual and physical abuse and substance use problems. A review. Clin Psychol Rev 2002; 22: $27-77$

${ }^{4}$ Ouimette P, Brown PJ. Trauma and substance abuse. Causes, consequences, and treatment of comorbid disorders. Washington, DC: American Psychological Association, 2003

${ }^{5}$ Ouimette PC, Ahrens C, Moos RH et al. Posttraumatic stress disorder in substance abuse patients: Relationship to 1-year posttreatment outcomes. Psychology Addict Behaviors 1997; 11: 34-47

${ }^{6}$ Najavits LM, Weiss RD, Shaw SR. The link between substance abuse and posttraumatic stress disorder in women. A research review. Am J Addict 1997; 6: 273-283

${ }^{7}$ Schneider U, Altman A, Baumann M et al. Comorbid anxiety and affective disorder in alcohol-dependent patients seeking treatment: The first multicenter study in Germany. Alcohol Alcoholism 2001; 36: $219-223$

${ }^{8}$ De Bernardo GL, Newcomb M, Toth A et al. Comorbid psychiatric and alcohol abuse/dependence disorders: Psychosocial stress, abuse, and personal history factors of those in treatment. J Addict Diseases 2002; 21: $43-59$

${ }^{9}$ Landheim AS, Bakken K, Vaglum P. Gender differences in the prevalence of symptom disorders and personality disorders among polysubstance abusers and pure alcoholics. Eur Addict Res 2003; 9: 8-17

${ }^{10}$ Verheul R, Hartgers C, Van den Brink W et al. The effect of sampling, diagnostic criteria and assessment procedures on the observed prevalence of DSM-III-R personality disorders among treated alcoholics. J Stud Alcohol 1998; 59 (2): 227-236

${ }^{11}$ Langeland W, Van den Brink W, Draijer N et al. Sensitivity of the Addiction Severity Index physical and sexual assault items: Preliminary findings on gender differences. Eur Addict Res 2001; 7: 193 - 197

12 Langeland W, Van den Brink W, Draijer N. Trauma, trauma-related distress, and perceived parental dysfunction: Associations with severity of drinking problems in treated alcoholics. J Nerv Ment Disease 2002; 190: $337-340$

${ }^{13}$ Langeland W, Draijer N, Van den Brink W. Trauma and dissociation in treatment-seeking alcoholics: Towards a resolution of inconsistent findings. Compreh Psychiatry 2002; 43: 195-203

${ }^{14}$ Langeland W, Draijer N, Van den Brink W. Assessment of lifetime physical and sexual abuse in treated alcoholics. Validity of the Addiction Severity Index. Addict Behaviors 2003; 28: 871 - 881
${ }^{15}$ Langeland W, Draijer N, Van den Brink W. Psychiatric comorbidity in treatment-seeking alcoholics: The role of childhood trauma and perceived parental dysfunction. Alcoholism: Clin Exp Res 2004; 28 : $441-447$

${ }^{16}$ Draijer N. Structured Trauma Interview. Amsterdam: Department of Psychiatry, Vrije University, 1989

${ }^{17}$ Windle M, Windle RC, Scheidt DM et al. Physical and sexual abuse and associated mental disorders among alcoholic inpatients. Am J Psychiatry 1995; 152: $1322-1328$

18 Pollock VE, Briere J, Schneider L et al. Childhood antecedents of antisocial behavior: Parental alcoholism and physical abusiveness. Am J Psychiatry 1990; 147: 1290 - 1293

19 Johnson JG, Cohen P, Brown J et al. Childhood maltreatment increases risk for personality disorders during early adulthood. Arch Gen Psychiatry 1999; 56: 600-606

${ }^{20}$ Yates WR, Petty F, Brown K. Alcoholism in males with antisocial personality disorder. Int J Addictions 1988; 23: 999-1010

${ }^{21}$ Van der Kolk BA, Fisler RE. Childhood abuse and neglect and loss of self-regulation. Bull Menninger Clin 1994; 58: 145-168

22 Ford JD, Kidd P. Early childhood trauma and disorders of extreme stress as predictors of treatment outcome with chronic posttraumatic stress disorder. J Trauma Stress 1998; 11: 743 - 761

${ }^{23}$ Allen JG, Huntoon J, Evans RB. Complexities in complex posttraumatic stress disorder in inpatient women: evidence from cluster analysis of MCMI-II Personality Disorder Scales. J Pers Assess 1999; 73: 449-471

24 Roth S, Newman E, Pelcovitz D et al. Complex PTSD in victims exposed to sexual and physical abuse: Results from the DSM-IV field trials for posttraumatic stress disorder. J Trauma Stress 1997; 10: 539-555

${ }^{25}$ Alarcon RD, Glover SG, Deering CG. The cascade model: An alternative to comorbidity in the pathogenesis of posttraumatic stress disorder. Psychiatry 1999; 62: 114-124

${ }^{26}$ Herman JL. Complex PTSD: A syndrome in survivors of prolonged and repeated trauma. J Trauma Stress 1992; 5: 377-391

${ }^{27}$ Carlson EB. Trauma assessments. A clinician's guide. New York: The Guilford Press, 1997

${ }^{28}$ Deering CG, Glover SG, Ready D et al. Unique patterns of comorbidity in posttraumatic stress disorder from different sources of trauma. Compreh Psychiatry 1996; 37: 336-346

${ }^{29}$ Rothbaum BO, Foa EB, Riggs DS et al. A prospective evaluation of posttraumatic stress disorder in rape victims. J Trauma Stress 1992; 5: $455-475$

30 Orsillo SM, Heimberg RG, Juster HR et al. Social phobia and PTSD in Vietnam veterans. J Trauma Stress 1996; 9: 235 - 252

${ }^{31}$ Najavits LM. Seeking Safety: A new psychotherapy for posttraumatic stress disorder and substance use disorder. In: Ouimette PC, Brown P (Hrsg). Trauma and substance abuse: Causes, consequences, and treatment for comorbid disorders. Washington, DC: American Psychological Association, 2003: 147-169

32 Coffey SF, Dansky BS, Brady KT. Exposure-based, trauma-focused therapy for comorbid posttraumatic stress disorder-substance use disorder. In: Ouimette PC, Brown P (Hrsg). Trauma and substance abuse: Causes, consequences, and treatment for comorbid. Washington, DC: American Psychological Association, 2003: 127-146

${ }^{33}$ Chu JA. Rebuilding shattered lives. The responsible treatment for complex posttraumatic and dissociative disorders. New York: John Wiley \& Sons, 1998 